\title{
The ferric yersiniabactin uptake receptor FyuA is required for efficient biofilm formation by urinary tract infectious Escherichia coli in human urine
}

Correspondence

Per Klemm

pkl@biocentrum.dtu.dk

Received 24 July 2007

Revised 26 September 2007

Accepted 28 September 2007

\author{
Viktoria Hancock, Lionel Ferrières and Per Klemm
}

\author{
Microbial Adhesion Group, BioCentrum-DTU, Technical University of Denmark, DK-2800 Lyngby, \\ Denmark
}

\begin{abstract}
Urinary tract infection (UTI) is the most common infection in patients with indwelling urinary catheters, and bacterial biofilm formation is a major problem in this type of infection. Escherichia coli is responsible for the large majority of UTIs. Free iron is strictly limited in the human urinary tract and there is fierce competition between the host and infectious bacteria for this essential metal. Urinary tract infectious $E$. coli have highly efficient mechanisms of iron acquisition, one of which is the yersiniabactin system. The fyuA gene, encoding the yersiniabactin receptor, is one of the most upregulated genes in biofilm; it was upregulated 63-fold in the E. coli UTI strain VR50. FyuA was found to be highly important for biofilm formation in iron-poor environments such as human urine. Mutants in fyuA show aberrant biofilm formation and the cells become filamentous; a VR50fyuA mutant showed a $92 \%$ reduction in biofilm formation in urine flow-cell chambers compared with the wild-type. The FyuA/yersiniabactin system is known to be important for virulence. Here we demonstrate a direct link between FyuA and biofilm formation in iron-poor environments. We also show that the availability of iron greatly influences UTI strains' ability to form biofilm.
\end{abstract}

\section{INTRODUCTION}

Iron is essential for bacterial growth. Bacteria face ironlimiting conditions in the mammalian host, where free iron is strictly limited and iron is normally bound to sequestering proteins such as transferrin and lactoferrin. To counter such iron-limiting conditions bacteria use different highly efficient mechanisms of iron acquisition. A typical high-affinity iron-uptake system consists of a lowmolecular-mass $\mathrm{Fe}^{3+}$-chelating compound, known as a siderophore, combined with its cognate membrane-located receptor (Martinez et al., 1990). Such iron-acquisition systems are generally regarded as important virulence or fitness factors. A range of enterobacteria contain a gene cluster called the high-pathogenicity island (HPI) encoding proteins for biosynthesis of the yersiniabactin (Ybt) siderophore and its uptake system (Rakin et al., 1999; Schubert et al., 1998, 2004). The HPI is widespread among members of the Enterobacteriaceae and is essential for virulence in Yersinia and certain pathotypes of Escherichia coli (Schubert et al., 2004). One of the important genes residing on the HPI is fyuA, encoding the $71 \mathrm{kDa}$

Abbreviations: $\mathrm{HPI}$, high-pathogenicity island; UPEC, uropathogenic $E$. coli; UTI, urinary tract infection.

The microarray data for this paper have been deposited in ArrayExpress (http://www.ebi.ac.uk/arrayexpress) with accession number E-MEXP926. outer-membrane protein FyuA (ferric yersiniabactin uptake), which act as a receptor for Fe-Ybt siderophore uptake (Heesemann et al., 1993; Rakin et al., 1994; Schubert et al., 2002). The fyuA gene has been associated with virulence in many members of the Enterobacteriaceae (Schubert et al., 1999, 2002).

Urinary tract infection (UTI) is a serious health problem that affects millions of people each year (Stamm \& Norrby, 2001). The recurrence rate is high, and often the infections are particularly troublesome and become chronic with multiple episodes. UTI usually starts as a bladder infection but often ascends to affect the kidneys and ultimately can result in renal failure or dissemination to the blood. UTI is the most common infection in patients with indwelling bladder catheters, and bacteriuria is essentially unavoidable in this patient group (Foxman, 2002). We have recently found that two phenotypic traits are important and contribute to the urinary tract-colonizing properties of many UTI strains, namely fast growth and superior biofilm formation in urine (Hancock et al., 2007; Roos et al., 2006a, b).

Rather than existing as individual planktonic cells, most bacteria generally live attached to surfaces as sessile communities, also referred to as biofilms (Costerton et al., 1995, 1999). Biofilms are commonly associated with many health problems (Costerton et al., 1999; O'Toole et al., 2000) and can form on virtually any type of surface. Bacterial biofilms have attracted particular attention, 
because many persistent and chronic bacterial infections are now believed to be linked to the formation of biofilms. Also, virtually all medical implants, such as indwelling urinary catheters, are prone to colonization by bacteria and the resultant biofilms often serve as a source for recurrent infections. Bacterial biofilm infections are particularly problematic, because sessile bacteria can withstand host immune defence mechanisms and are extremely resistant to antibiotics, biocides and hydrodynamic shear forces that can efficiently clear corresponding planktonic bacteria (Costerton et al., 1995, 1999). In the urinary tract bacterial biofilm-associated infections include chronic cystitis, prostatitis and catheter- and stent-associated infections (Warren, 2001). On this background we have analysed the role of FyuA-mediated iron acquisition and of iron concentration on biofilm formation of UTI E. coli strains in human urine.

\section{METHODS}

Bacterial strains and media. Strains VR50, 83972 and VR89 are asymptomatic bacteriuria (ABU) E. coli strains (Roos et al., 2006a). J96, 536 and NU14 are three uropathogenic E. coli (UPEC) strains isolated from severe cases of UTI. Pooled human urine, or MOPS minimal medium supplemented with $0.2 \%$ glucose (Neidhardt et al., 1974), were used in all experiments. Human urine was collected from a pool of 15 healthy men and women volunteers who had no history of UTI or antibiotic use in the prior 2 months. For each experiment, urine was collected randomly from three to five of these individuals ( $\mathrm{pH}$ 5.5-7.5). The urine was pooled ( $\mathrm{pH}$ 6.5-7.0), filter-sterilized, stored at $4{ }^{\circ} \mathrm{C}$, and used within the following 2-3 days. In the iron experiment, the urine was supplemented with either $10 \mu \mathrm{M} \mathrm{FeCl}_{3}$ or $200 \mu \mathrm{M} 2,2^{\prime}$-dipyridyl (iron chelator).

Microarray analysis. The microarray results of the yersiniabactin HPI cluster are extracted from a previously published complete genome microarray study of E. coli strains 83972 and VR50 during biofilm formation (Hancock \& Klemm, 2007). GeneChip E. coli Genome 2.0 Arrays (Affymetrix) were used for hybridization of the labelled cDNA. For VR50, three chips were hybridized with samples grown in three individual flasks in MOPS medium, three chips were hybridized with samples from cells grown in pooled human urine in three individual flasks, and three chips were hybridized with samples from biofilm cells grown in pooled human urine in three individual Petri dishes. The nine VR50 arrays were normalized together and fold changes during biofilm growth in urine compared with planktonic growth in MOPS and in urine were calculated; the three arrays hybridized with samples from biofilm growth on Petri dishes were compared with the three arrays hybridized with samples from planktonic growth (i) in MOPS and (ii) in urine. In a third comparison, fold changes were calculated by comparing the arrays hybridized with samples from planktonic urine with planktonic MOPS. The supporting microarray data have been deposited in ArrayExpress (http://www.ebi.ac.uk/arrayexpress) with accession number E-MEXP-926.

Construction of fyuA knockout mutants. FyuA mutants of VR50 and 83972 were constructed using the $\lambda$ Red recombinase gene replacement system (Datsenko \& Wanner, 2000). Briefly, the $n p t$ gene from plasmid pKD4 was amplified using a primer set containing $40 \mathrm{nt}$ homology extensions of the fyuA target gene: P707 (5'-CGCCGTCTTACAGGGACTCACAACAATGAAAATGACACGGGTGTAGGCTGGAGCTGCTTCG-3') and P708 (5'-ATCAGAAGAAATCAATTCGCGTATTGATACCGACGGTGCGCATATGAATATCCTCCT-
TAG-3'). The PCR product was transformed into host strains containing pKD46, and kanamycin-resistant colonies were selected. The $\lambda$ Red helper plasmid pKD46 was cured by growth at $37^{\circ} \mathrm{C}$ and the correct double-crossover was confirmed by PCR (P711, 5'CGCCGTCTTACAGGGACTC-3'; P712, 5'-CAATTCGCGTATTGATACC-3').

Construction of fyuA plasmid pVR3. The fyuA gene of VR50 was amplified by PCR (P713, 5' -GCGCCAAGCTTGTCGACCGTTATCGCC- ${ }^{\prime}$ and P714, 5' -GCGCCGGATCCCGACTTTGCTGAGTTACTGG-3') containing HindIII and BamHI restriction sites, and cloned into pBR322. The resulting plasmid, pVR3, was transformed into VR50, VR50fyuA, MG1655 $\Delta$ fim $\Delta$ flu and the three UPEC strains J96, 536 and NU14.

Biofilm formation in microtitre plates. Cells were pre-grown in pooled human urine and $10 \mu \mathrm{l}$ aliquots were used to inoculate $1 \mathrm{ml}$ urine in 24-well flat-bottom microplates (Iwaki). The microplates were incubated statically at $37^{\circ} \mathrm{C}$ overnight and biofilm was monitored by crystal violet staining as described previously (Hancock et al., 2007). Each strain was assayed in three or four wells on each plate and all experiments were repeated at least five times in different batches of urine. Two to four wells were used as blanks containing sterile urine (with metal, chelator or antibiotics when appropriate) and three or four wells were inoculated with wild-type strain for reference.

Carrying capacity and growth rate determination. Cells were grown in glass tubes containing $2 \mathrm{ml}$ human urine (statically or shaking at 130 r.p.m.) and in microtitre wells statically containing $1 \mathrm{ml}$ urine at $37^{\circ} \mathrm{C}$ for $16 \mathrm{~h}$. After overnight incubation the medium in the microtitre wells was pipetted thoroughly up and down to disperse the biofilm and the final $\mathrm{OD}_{600}$ was measured. Each strain was assayed in three tubes and four wells in each experiment and the whole experiment was repeated three times in different batches of urine. For growth rate determination, each strain was grown in triplicate in $25 \mathrm{ml}$ urine and $\mathrm{OD}_{600}$ was measured every $30 \mathrm{~min}$ until stationary phase was reached.

Biofilm formation in flow-cell chambers. Flow-chamber experiments were performed at $37{ }^{\circ} \mathrm{C}$ in human urine, essentially as described previously (Christensen et al., 1999). The cells were allowed to attach to the substratum for $1 \mathrm{~h}$ before the flow $\left(3 \mathrm{ml}\right.$ urine $\mathrm{h}^{-1}$ ) was turned on. Biofilm formation was monitored by scanning confocal laser microscopy (SCLM; Zeiss LSM510 microscope) using a $40 \times / 1.3$ Plan-Neofluar oil objective. The biofilm structure was visualized by fluorescent staining with SYTO9. Each strain was inoculated in duplicate channels and the whole experiment was repeated twice in different batches of urine. Biomass and percentage substratum coverage were determined using COMSTAT image-processing software (Heydorn et al., 2000); Z-stacks were collected from five randomly selected regions from each independent biofilm.

\section{RESULTS}

\section{Upregulation of fyuA and other HPI-associated genes}

E. coli strain VR50 is a clinical urinary tract isolate; it grows well in pooled human urine and is an excellent biofilm former in this growth medium (Hancock et al., 2007; Roos et al., 2006a). We have recently studied the global gene expression in this strain during growth in biofilm 
(Hancock \& Klemm, 2007). The genomic expression profile of E. coli VR50 obtained during biofilm formation in urine was compared with that during exponential growth in minimal (MOPS-glucose) medium and pooled human urine. Here we focus on iron uptake genes differentially expressed in biofilm compared with planktonic growth (Fig. 1). The $c 2436$ gene shares $99.9 \%$ amino acid identity with the $f y u A$ gene, encoding the ferric yersiniabactin uptake receptor, which has been associated with virulence in extraintestinal pathogenic E. coli
(Schubert et al., 2002, 2004). The fyuA gene (c2436) and its flanking genes, c2418-c2437, were among the most upregulated in VR50 biofilm (3.1-57-fold compared with urine) and this cluster, i.e. HPI, was by far the largest contiguous upregulated gene cluster during biofilm growth. The genes in this cluster are homologous to the genes encoding Ybt biosynthesis (c2419, c2424-c2434), transport (c2421 and 2422) and the transcriptional regulator (c2423). The expression of the biosynthetic and transport genes is repressed by iron and the ferric uptake
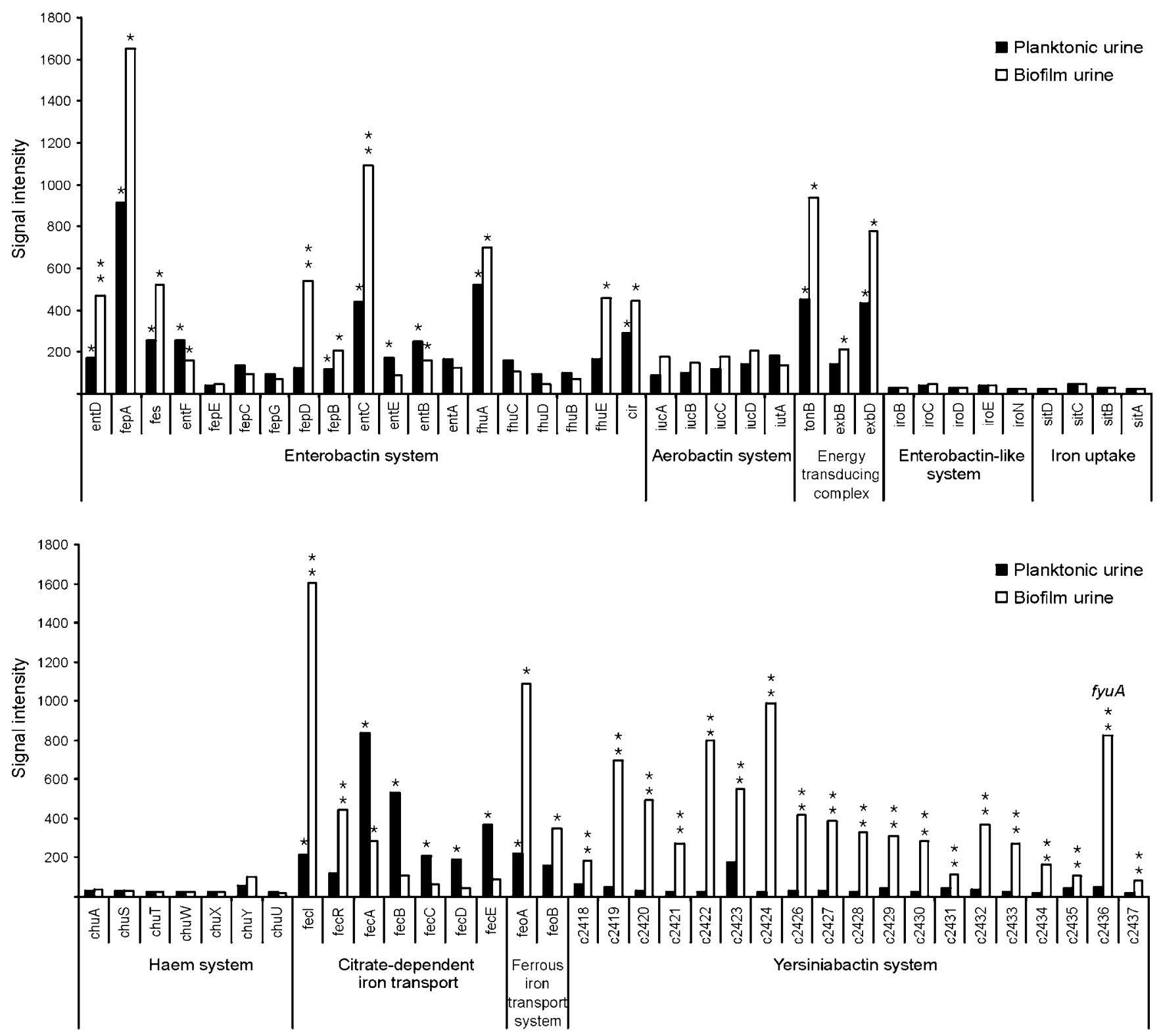

Fig. 1. Signal intensity of iron acquisition and uptake genes of the E. coli VR50 UTI strain during planktonic and biofilm urine growth. The yersiniabactin system of the HPI (c2418-c2437) was the only iron system upregulated during biofilm growth compared with planktonic growth in urine. c2422, c2424 and c2436 (fyuA) were among the ten highest upregulated genes in biofilm. *Upregulation compared with planktonic growth in MOPS minimal media. ${ }^{\star}$ Upregulation compared with planktonic growth in both MOPS and urine. 
regulator Fur (Schubert et al., 2004). c2422 and fyuA (c2436) were respectively the second- and the fifth-highest induced genes overall during biofilm formation, and moreover, seven of the 19 genes in this cluster belonged to the 20 most upregulated in biofilm compared with planktonic growth in urine. The upregulation of these 19 genes in urine biofilm was significant compared with planktonic growth both in urine and in minimal medium, indicating a role specific for the biofilm growth mode and not dependent on urine composition. The HPI was the only iron-acquisition system upregulated in urine biofilm that was not upregulated in planktonic urine compared with MOPS minimal medium (Fig. 1). The high upregulation of this gene cluster indicates that these genes encoding biosynthesis and uptake of yersiniabactin may play a role during biofilm growth.

\section{An fyuA-null mutant shows significantly reduced biofilm-forming capacity}

By virtue of its high expression level during biofilm formation we surmised that the $f y u A$ gene could play an important role during sessile growth. A deletion mutant, VR50fyuA, was made that carried a lesion of the central region of the $f y u A$ gene encompassing $97 \%$ of the gene. Growth experiments with VR50fyuA in pooled human urine revealed no growth limitations since the mutant strain reached the same final cell density as the wild-type parent, during both static and shaking growth conditions, and no significant difference in growth rate could be observed $\left(0.0247 \pm 0.0023\right.$ and $0.0259 \pm 0.0016 \mathrm{~min}^{-1}$ for VR50 and VR50fyuA, respectively, Student's $t$ test, $P=0.49$ ) (Fig. 2c). However, the knockout mutant showed significantly reduced biofilm formation capacity in microtitre plates (Fig. 2a). The FyuA status of the mutant strain was restored by cloning of the fyuA gene, resulting in plasmid pVR3, and subsequent introduction of pVR3 into VR50fyuA. Strain VR50fyuA harbouring plasmid pVR3 performed essentially like the VR50 parent with respect to biofilm formation (Fig. 2a).

\section{The fyuA deletion mutant shows reduced biofilm formation in urine flow chambers}

The urinary tract is a highly hydrodynamic environment with a constant flow of urine that could affect the way biofilms develop for example on artificial medical implants such as indwelling urinary catheters. Therefore, in order to mimic more realistic conditions, we investigated the ability of the fyuA mutant to form biofilm in a flow-chamber system using human urine as growth medium. VR50 and VR50fyuA were inoculated in flow chambers and the biofilm formation on glass slides was monitored over time by confocal microscopy. It turned out that, in line with the results obtained in microtitre plates, the mutant strain VR50fyuA was significantly impaired in biofilm formation both with respect to biomass and also with respect to biofilm structure in the flow-chamber system compared with the wild-type strain (Fig. 3). Already after $16 \mathrm{~h}$, the wild-type strain had colonized a larger proportion of the surface compared with the fyuA mutant. After $24 \mathrm{~h}$ and $40 \mathrm{~h}$, the wild-type strain had covered the entire surface, while the fyuA mutant did not show any improvement in biofilm formation - even after $40 \mathrm{~h}$ it had only developed small, scattered patches on the surface. Close inspection revealed that the morphology of biofilm-associated VR50fyuA cells deviated significantly from that of the parent: the cells were elongated and snake-like in appearance (Fig. 3). Density scanning of the biofilms at $40 \mathrm{~h}$ revealed that the biofilm surface coverage and biofilm volume of the fyuA mutant were $15 \%$ and $8 \%$, respectively, of that of the wild-type $(P<0.001)$. Altogether, these results demonstrate that FyuA is required for normal biofilm formation of strain VR50 under hydrodynamic urine flow conditions, mimicking the conditions encountered in the urinary tract.

\section{The fyuA-null-associated reduction in biofilm-forming capacity can be observed in other UTI E. coli}

The well-characterized UTI E. coli strain 83972 is an excellent biofilm-former in human urine (Hancock et al., 2007). Strain 83972 seems to employ several systems for iron acquisition when grown in urine. Global gene expression profiling has revealed upregulation of virtually all E. coli iron-acquisition systems when grown both in vitro and in vivo; the genes encoding enterobactin, siderophore, aerobactin and salmochelin systems, i.e. fep, ent, fhu, iutA, iuc and iro, as well as other iron-transport systems such as the sit and chu clusters, were all significantly induced in urine (Roos \& Klemm, 2006; Roos et al., 2006b). Also the fyuA gene was seen to be upregulated up to 25-fold in the human urinary tract (Roos \& Klemm, 2006). In this study, a deletion mutant, $83972 f y u A$, showed a $53 \%$ reduction in biofilm-forming capability in microtitre plates in human urine as compared with the parent strain (Fig. 2a). The result demonstrates that the fyuA yersiniabactin receptor-encoding gene is required for efficient biofilm formation by strain 83972 in human urine, despite the many other iron-acquisition systems expressed by the strain, and that importance of FyuA in biofilm formation is not specific for strain VR50.

\section{Iron concentration affects biofilm formation}

FyuA is the outer-membrane receptor of the Fe-Ybt siderophore, importing iron into the cells (Schubert et al., 2004). The iron concentration in urine is limited, restricting bacterial activity in this growth medium. However, the ability of a UTI strain to form biofilm in urine is not directly correlated with its growth rate or carrying capacity in urine (Ferrières et al., 2007; Hancock et al., 2007). Therefore, three E. coli UTI strains were tested for biofilm formation in urine with added iron chelator at chelator concentrations that did not affect final cell 

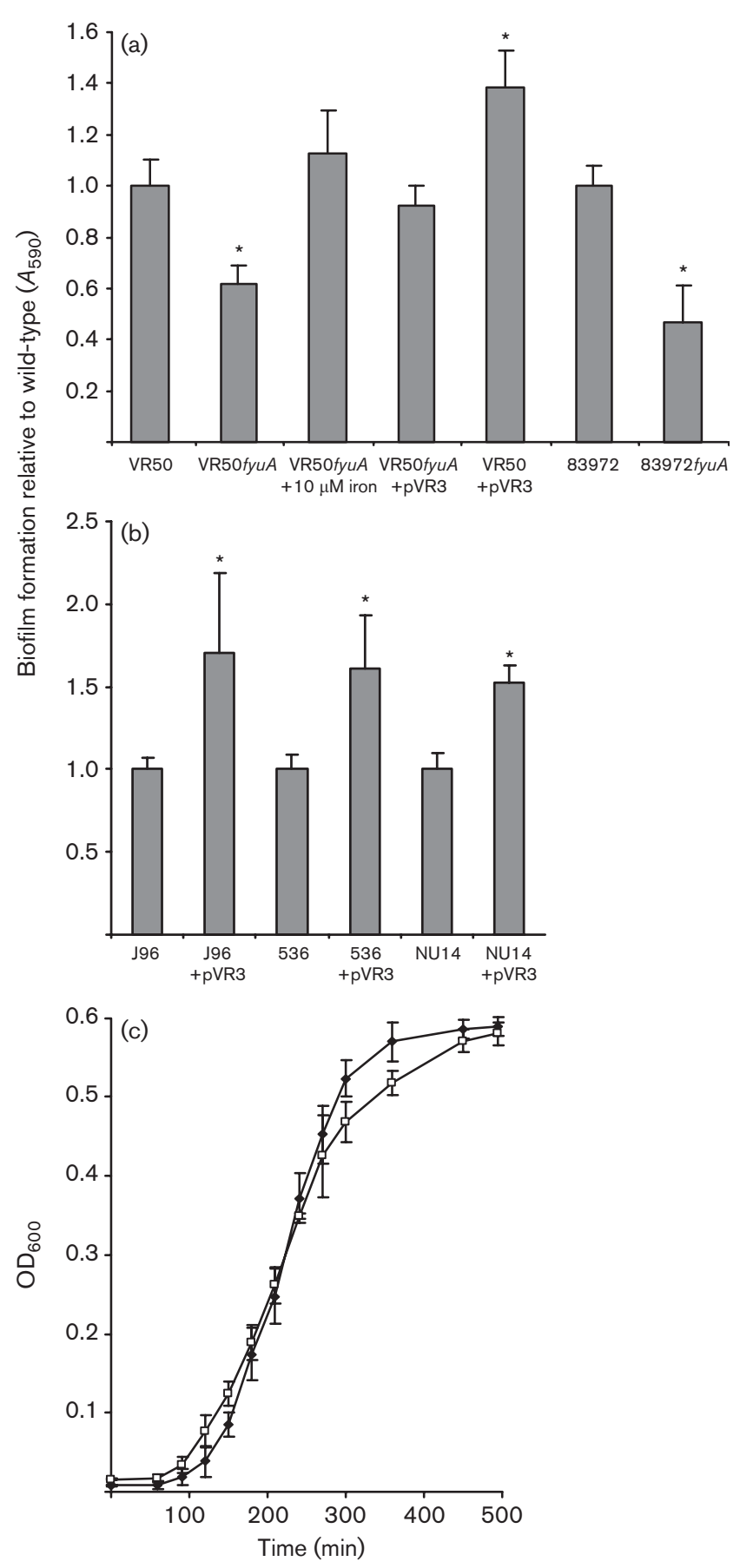

densities in liquid cultures. Also, biofilm formation was tested in urine with added iron. It transpired that strains 83972, VR50 and VR89 all formed significantly less biofilm in the presence of iron chelator and more biofilm with iron added to the growth medium (Fig. 4). Furthermore, the reduced biofilm formation observed for the mutant strain VR50fyuA could be relieved by addition of iron to the growth medium (Fig. 2a), indicating that the presence of FyuA becomes less important when the availability of iron is higher. Additionally, biofilm formation of the mutant
Fig. 2. FyuA influences the biofilm-forming capacity of UTI strains in microtitre plates in human urine. (a) Biofilm formation was reduced in the fyuA mutant of the UTI isolate VR50. The biofilmforming capacity could be regained by the addition of iron to the growth medium or by complementation with a plasmid expressing the fyuA gene (pVR3). Biofilm formation was also reduced in an fyuA mutant of the UTI strain 83972. ${ }^{*} P<0.001$. (b) Overexpression of the fyuA gene (pVR3) by J96, 536 and NU14 significantly increased biofilm formation of these three UPEC strains. ${ }^{*} P<0.01$. All experiments (a) and (b) were performed in pooled human urine and repeated at least five times in different urine batches; error bars indicate standard deviation of five or more independent experiments. (c) Representative growth curves of VR50 ( $)$ and the mutant VR50fyuA $(\square)$ in human urine; error bars indicate standard deviation of three shake flasks.

strain VR50fyuA in urine with added chelator was reduced by $72 \%$ compared with the parent in the same chelatorcontaining medium (Student's $t$ test, $P<0.001$ ), which corresponds to a reduction almost twice as large as in unmodified urine (i.e. 38\%). This indicates that the importance of FyuA in biofilm formation increases when iron becomes limited. Altogether, the results indicate that availability of iron is critical and influences the ability to form biofilm - the biofilm formation in urine increased with increasing iron concentration for a number of UTI strains, and the expression of FyuA during biofilm formation in human urine becomes more important as the availability of iron decreases.

\section{Introduction of an fyuA-encoding plasmid increases biofilm formation of UPEC strains}

Our results showed that an increased iron concentration resulted in better biofilm formation; we therefore wanted to investigate whether overexpression of fyuA by virtue of increased copy number would result in increased biofilm formation induced by increased iron uptake. When strain VR50 was transformed with pVR3 containing the fyuA gene a $38 \%$ increase in biofilm formation was observed (Fig. 2a). Transformation of three well-characterized UPEC strains (which all carry the HPI), J96, 536 and NU14, with pVR3 revealed a significant increase in biofilm formation in human urine; J96, 536 and NU14 showed 70\%, 61\% and $52 \%$ higher biofilm formation, respectively (Fig. 2b). This result indicates that iron limitation can be overcome via overexpression of an efficient iron-uptake system such as FyuA. Consequently, when UPEC strains J96, 536 and NU14 are engineered to take up iron more efficiently they are also able to produce biofilms better, despite the plasmid burden.

\section{The FyuA protein itself does not account for improved biofilm formation}

FyuA is located in the outer membrane. The FyuA protein per se could theoretically contribute to biofilm formation by altering the adhesive characteristics of the cell - for 

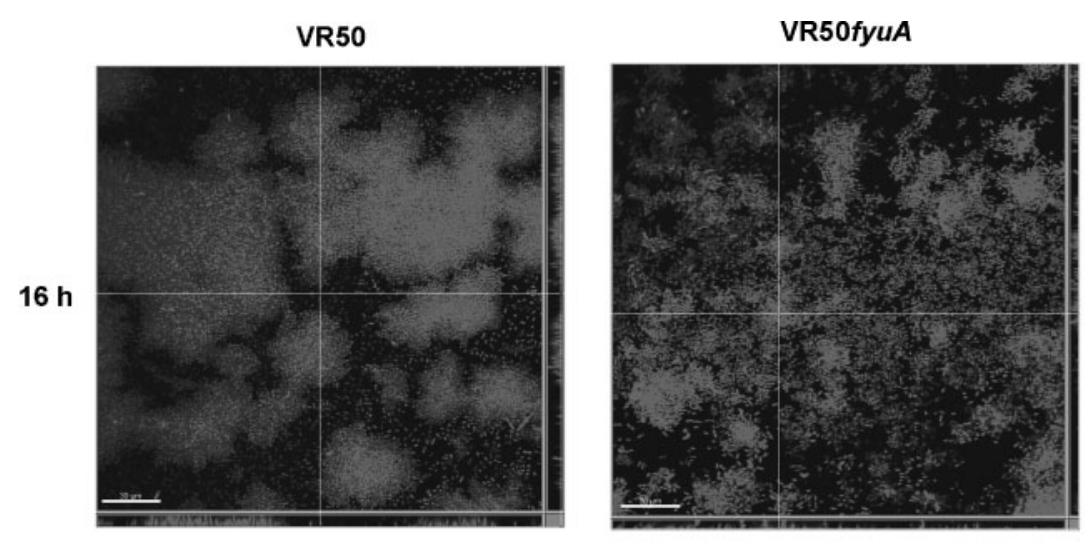

$24 \mathrm{~h}$
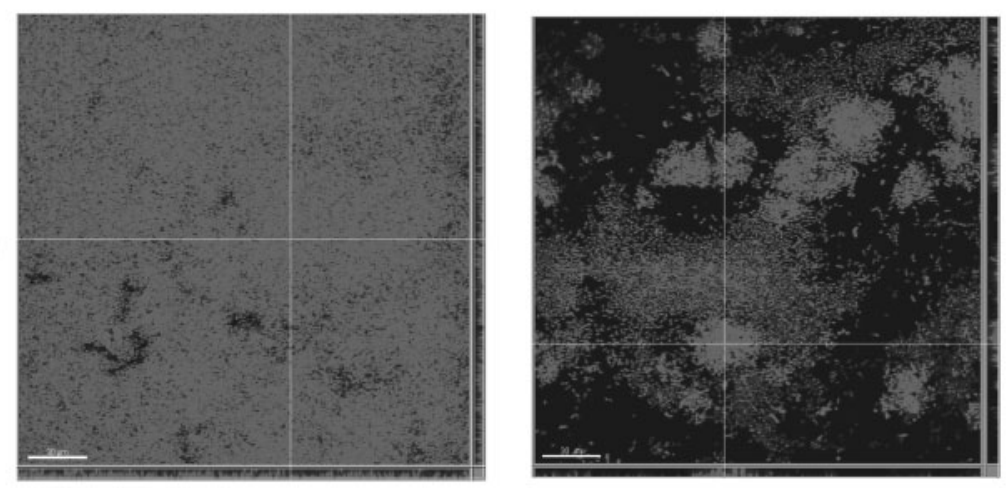

$40 \mathrm{~h}$

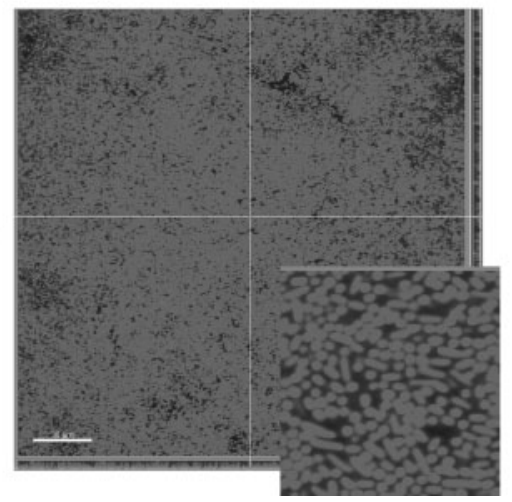

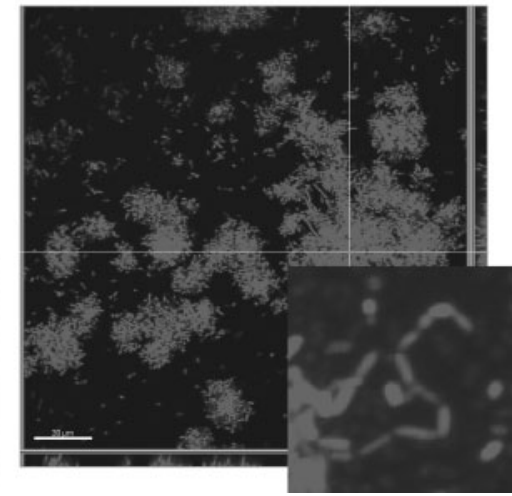

Fig. 3. Time-course of biofilm formation by VR50 and VR50fyuA grown in flow chambers in urine. The fyuA-null mutant showed strongly reduced biofilm formation compared with the wild-type in the flow-chamber system. Biofilm development was monitored by scanning confocal laser microscopy at the indicated time points after inoculation. Vertical crosssections through the biofilm were generated and simulated $x z$ and $y z$ vertical cross-sections are presented. Scale bars, $30 \mu \mathrm{m}$. example by inducing autoaggregation, which is known to improve biofilm formation (Kjaergaard et al., 2000; Schembri et al., 2002). Therefore, a mutant version of MG1655 incapable of producing the surface elements Ag43 and type 1 fimbriae was complemented with the fyuA plasmid and studied with regard to biofilm formation and cell aggregation. Like other K-12 strains, MG1655 does not carry the HPI. Cells were grown under conditions (LB medium) where iron was not growth limiting. No improved biofilm formation in microtitre plates (Student's $t$ test, $P=0.45$ ) and no cell aggregation could be observed for the FyuA-expressing cells compared with wild-type cells. Furthermore, cell aggregation properties of VR50, VR50fyuA, VR50fyuA(pVR3) and VR50(pVR3), after growth in urine and $\mathrm{LB}$, were compared by settling curves and light microscopy. No differences in settling rates and no cell aggregation were observed. The same results were seen when comparing the three UPEC strains (J96, 536 and NU14) with their respective plasmid-containing counterpart. Arguably, it is unlikely that the FyuA protein itself contributes to enhanced biofilm formation due to any autoaggregation property and independent of iron uptake.

\section{DISCUSSION}

More than $50 \%$ of all bacterial microbial infections are associated with the formation of biofilms, and artificial medical implants are particularly prone to biofilm formation (Costerton et al., 1999). In the case of a highflow environment like the urinary tract, biofilm-associated infections are particularly important, notably in infections 


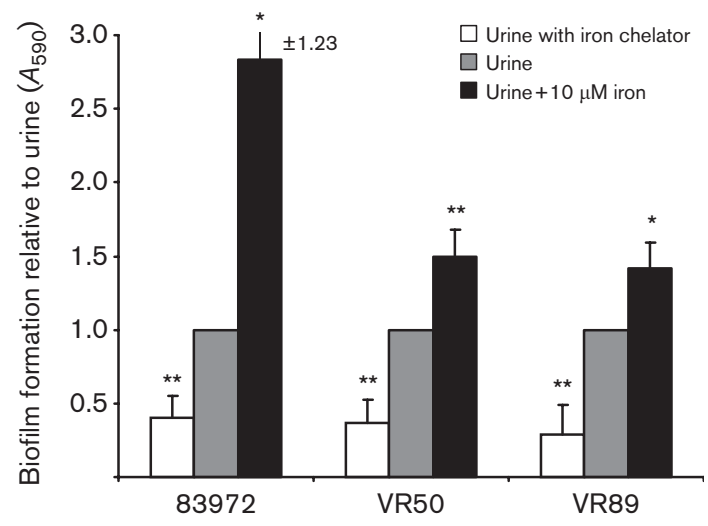

Fig. 4. The availability of iron greatly influences biofilm formation of the three UTI strains 83972, VR50 and VR89 in microtitre plates in human urine. Addition of an iron chelator $\left(100 \mu \mathrm{M} 2,2^{\prime}\right.$-dipyridyl) or addition of iron $(10 \mu \mathrm{M} \mathrm{FeCl})$ to the growth medium significantly changes the biofilm-forming capacity in urine. ${ }^{\star} P<0.02,{ }^{* \star} P<0.001$. The experiment was performed in pooled human urine and repeated at least five times in different batches of urine; error bars indicate standard deviation of five or more independent experiments.

associated with urinary catheters and stents, where biofilms cause flow obstruction and serve as a source for recurrent infections (Foxman, 2002; Morris et al., 1999; Reid et al., 1992). However, mounting evidence supports the notion that biofilm formation also is involved in non-implanttype UTIs (Anderson et al., 2003; Kumon, 2000; Soto et al., 2006).

Iron is essential for both bacteria and humans, and for most infectious bacteria there is intense competition for iron with the host. The concentration of soluble forms of iron is very low in the urinary tract and is known to limit bacterial activity (Roos \& Klemm, 2006; Russo et al., 1999; Snyder et al., 2004). Here we show that the ferric yersiniabactin uptake receptor FyuA is important for biofilm formation by urinary tract infectious E. coli. Our experimental conditions, using pooled human urine for biofilm formation in flow chambers, closely mimic catheterized patients. Several lines of evidence indicated the importance of the FyuA Ybt-Fe uptake receptor during biofilm formation. Firstly, global gene expression profiling of UTI strain VR50 during biofilm formation in urine showed that all genes located in the HPI were significantly upregulated compared with planktonic cells; interestingly, this was the only iron-acquisition system displaying upregulation in biofilm urine growth compared with planktonic urine that was not upregulated in planktonic urine compared with MOPS minimal medium (Fig. 1). The gene encoding the FyuA homologue was among the highest upregulated of all genes (63-fold). Secondly, an fyuA mutant showed significant reduction in biofilm formation compared with its parent - both on polystyrene in microtitre plates and on glass in flow chambers. However, when complemented in trans or supplied with extra iron the mutant regained its biofilmforming faculty. Thirdly, introduction of an fyuA-encoding plasmid into the well-characterized UPEC strains, i.e. J96, 536 and NU14, with the net effect of increasing the copy number of fyuA and thereby enhancing iron uptake capability, increased biofilm formation significantly. The importance of FyuA in biofilm formation was not a unique trait of strain VR50 but was also seen in another strain, 83972, in which an fyuA-knockout mutation had a similar, severe, effect on biofilm formation.

The majority of infectious E. coli strains possess multiple systems for ferric ion uptake, notably the relatively lowaffinity aerobactin system and the two high-affinity systems yersiniabactin and enterobactin (Braun, 2003; Perry et al., 1999; Schubert et al., 1998). Our data support the notion that iron-limiting conditions affect biofilm-dwelling E. coli more than their planktonic counterparts. All genes located in the HPI were upregulated in urine biofilms compared with planktonic urine growth, whereas the aerobactin and enterobactin systems were not upregulated in urine biofilm compared with planktonic urine, indicating that iron uptake via yersiniabactin plays an important role in biofilm growth. HPI gene transcription is known to be strongly dependent on iron concentration (Fetherston et al., 1999); so even though urine contains some iron, cells in urine biofilms must be desperate for iron. Mutant strain VR50fyuA did not exhibit growth limitation during planktonic growth in urine compared with the VR50 parent, whereas it showed strongly reduced biofilm formation in the same batch of urine when compared with the parent. This is probably because the prevailing conditions in biofilms with high cell density result in even fiercer competition for iron and very low local concentrations of iron. Under such conditions high-affinity ironuptake systems like the yersiniabactin system become crucial. This does not rule out that other iron uptake systems might play a role in biofilms, but the array data indicate that the Ybt system is more 'biofilm specific' compared with other iron-related systems, which are upregulated during planktonic growth in urine as well.

The role of iron in biofilm formation has also been investigated in Pseudomonas aeruginosa, where iron depletion was shown to severely affect the shape and density of the biofilms (Banin et al., 2005). In P. aeruginosa intracellular iron concentration seems to serve as a signal in biofilm formation; disruption of iron-dependent processes inhibits growth and biofilm formation (Kaneko et al., 2007), and dispersal of biofilms can be induced by addition of iron chelators (Banin et al., 2006). We have not observed chelator-induced biofilm dispersal in our UTI E. coli biofilms and our data indicate that iron starvation per se is responsible for poor growth of biofilm-associated cells. Biofilms of VR50fyuA not only had significantly less biomass than the parent but morphology was also affected at the cell level: cell shape became filamentous compared with the parent. Interestingly, filamentous cell shape was recently reported to be involved in UTIs in the mouse 
model, and iron starvation might be involved in this phenomenon (Justice et al., 2006). Recently, the importance of FyuA was underlined in an experimental vaccine study targeting pathogenic extraintestinal E. coli; here FyuA turned out to be one of the top candidates (Durant et al., 2007).

Many bacterial diseases are associated with biofilm formation and there is mounting evidence that biofilm formation is also important in one of the top bacterial disease in humans, i.e. UTI, with more than 120 million cases per year globally. The biofilm mode of living is an important survival strategy or even in itself a virulence trait. It enhances bacterial resistance to hydrodynamic flow forces, to immune defences and to antibiotics. However, the biofilm mode of life comes at a price and in irondepleted environments like the human urinary tract iron starvation is a very real possibility. The HPI, encoding the yersiniabactin iron uptake system, has spread through the enteric group by horizontal gene transfer. Arguably, its success in terms of high incidence speaks of high selection pressure perhaps due to a requirement for biofilm formation in iron-depleted environments.

\section{ACKNOWLEDGEMENTS}

We thank Birthe Jul Jørgensen for expert technical assistance. This work was supported by grants from the Lundbeckfonden (271145971) and the Danish Medical Research Council (271-06-0555).

\section{REFERENCES}

Anderson, G. G., Palermo, J. J., Schilling, J. D., Roth, R., Heuser, J. \& Hultgren, S. J. (2003). Intracellular bacterial biofilm-like pods in urinary tract infections. Science 301, 105-107.

Banin, E., Vasil, M. L. \& Greenberg, E. P. (2005). Iron and Pseudomonas aeruginosa biofilm formation. Proc Natl Acad Sci U S A 102, 11076-11081.

Banin, E., Brady, K. M. \& Greenberg, E. P. (2006). Chelator-induced dispersal and killing of Pseudomonas aeruginosa cells in a biofilm. Appl Environ Microbiol 72, 2064-2069.

Braun, V. (2003). Iron uptake by Escherichia coli. Front Biosci 8, 1409-1421.

Christensen, B. B., Sternberg, C., Andersen, J. B., Palmer, R. J., Jr, Nielsen, A. T., Givskov, M. \& Molin, S. (1999). Molecular tools for study of biofilm physiology. Methods Enzymol 310, 20-42.

Costerton, J. W., Lewandowski, Z., Caldwell, D. E., Korber, D. R. \& Lappin-Scott, H. M. (1995). Microbial biofilms. Annu Rev Microbiol 49, 711-745.

Costerton, J. W., Stewart, P. S. \& Greenberg, E. P. (1999). Bacterial biofilms: a common cause of persistent infections. Science 284, 1318-1322.

Datsenko, K. A. \& Wanner, B. L. (2000). One-step inactivation of chromosomal genes in Escherichia coli K-12 using PCR products. Proc Natl Acad Sci U S A 97, 6640-6645.

Durant, L., Metais, A., Soulama-Mouze, C., Genevard, J.-M., Nassif, X. \& Escaich, S. (2007). Identification of candidates for a subunit vaccine against extraintestinal pathogenic Escherichia coli. Infect Immun 75, 1916-1925.
Ferrières, L., Hancock, V. \& Klemm, P. (2007). Biofilm exclusion of uropathogenic bacteria by selected asymptomatic bacteriuria Escherichia coli strains. Microbiology 153, 1711-1719.

Fetherston, J. D., Bertolino, V. J. \& Perry, R. D. (1999). YbtP and YbtQ: two ABC transporters required for iron uptake in Yersinia pestis. Mol Microbiol 32, 289-299.

Foxman, B. (2002). Epidemiology of urinary tract infections: incidence, morbidity, and economic costs. Am J Med 113 (Suppl. 1A), 5S-13S.

Hancock, V. \& Klemm, P. (2007). Global gene expression profiling of asymptomatic bacteriuria Escherichia coli during biofilm growth in human urine. Infect Immun 75, 966-976.

Hancock, V., Ferrières, L. \& Klemm, P. (2007). Biofilm formation by asymptomatic and virulent urinary tract infectious Escherichia coli strains. FEMS Microbiol Lett 267, 30-37.

Heesemann, J., Hantke, K., Vocke, T., Saken, E., Rakin, A., Stojiljkovic, I. \& Berner, R. (1993). Virulence of Yersinia enterocolitica is closely associated with siderophore production, expression of an iron-repressible outer membrane polypeptide of $65000 \mathrm{Da}$ and pesticin sensitivity. Mol Microbiol 8, 397-408.

Heydorn, A., Nielsen, A. T., Hentzer, M., Sternberg, C., Givskov, M., Ersboll, B. K. \& Molin, S. (2000). Quantification of biofilm structures by the novel computer program COMSTAT. Microbiology 146, 2395-2407.

Justice, S. S., Hunstad, D. A., Seed, P. C. \& Hultgren, S. J. (2006). Filamentation by Escherichia coli subverts innate defenses during urinary tract infection. Proc Natl Acad Sci U S A 103, 19884-19889.

Kaneko, Y., Thoendel, M., Olakanmi, O., Britigan, B. E. \& Singh, P. K. (2007). The transition metal gallium disrupts Pseudomonas aeruginosa iron metabolism and has antimicrobial and antibiofilm activity. J Clin Invest 117, 877-888.

Kjaergaard, K., Schembri, M. A., Ramos, C., Molin, S. \& Klemm, P. (2000). Antigen 43 facilitates formation of multispecies biofilms. Environ Microbiol 2, 695-702.

Kumon, H. (2000). Management of biofilm infections in the urinary tract. World J Surg 24, 1193-1196.

Martinez, J. L., Delgado-Iribarren, A. \& Baquero, F. (1990). Mechanisms of iron acquisition and bacterial virulence. FEMS Microbiol Rev 6, 45-56.

Morris, N. S., Stickler, D. J. \& McLean, R. J. C. (1999). The development of bacterial biofilms on indwelling urethral catheters. World J Urol 17, 345-350.

Neidhardt, F. C., Bloch, P. L. \& Smith, D. F. (1974). Culture medium for enterobacteria. J Bacteriol 119, 736-747.

O'Toole, G., Kaplan, H. B. \& Kolter, R. (2000). Biofilm formation as microbial development. Annu Rev Microbiol 54, 49-79.

Perry, R. D., Balbo, P. B., Jones, H. A., Fetherston, J. D. \& DeMoll, E. (1999). Yersiniabactin from Yersinia pestis: biochemical characterization of the siderophore and its role in iron transport and regulation. Microbiology 145, 1181-1190.

Rakin, A., Saken, E., Harmsen, D. \& Heesemann, J. (1994). The pesticin receptor of Yersinia enterocolitica: a novel virulence factor with dual function. Mol Microbiol 13, 253-263.

Rakin, A., Noelting, C., Schubert, S. \& Heesemann, J. (1999). Common and specific characteristics of the high-pathogenicity island of Yersinia enterolitica. Infect Immun 67, 5265-5274.

Reid, G., Denstedt, J. D., Kang, Y. S., Lam, D. \& Nause, C. (1992). Microbial adhesion and biofilm formation on ureteral stents in vitro and in vivo. $J$ Urol 148, 1592-1594.

Roos, V. \& Klemm, P. (2006). Global gene expression profiling of the asymptomatic bacteriuria Escherichia coli strain 83972 in the human urinary tract. Infect Immun 74, 3565-3575. 
Roos, V., Nielsen, E. M. \& Klemm, P. (2006a). Asymptomatic bacteriuria Escherichia coli strains: adhesins, growth and competition. FEMS Microbiol Lett 262, 22-30.

Roos, V., Ulett, G. C., Schembri, M. A. \& Klemm, P. (2006b). The asymptomatic bacteriuria Escherichia coli strain 83972 outcompetes UPEC strains in human urine. Infect Immun $\mathbf{7 4}$ 615-624.

Russo, T. A., Carlino, U. B., Mong, A. \& Jodush, S. T. (1999). Identification of genes in an extraintestinal isolate of Escherichia coli with increased expression after exposure to human urine. Infect Immun 67, 5306-5314.

Schembri, M. A., Givskov, M. \& Klemm, P. (2002). An attractive surface: gram-negative bacterial biofilms. Sci STKE 132, RE6.

Schubert, S., Rakin, A., Karch, H., Carniel, E. \& Heesemann, J. (1998). Prevalence of the "high-pathogenicity island" of Yersinia species among Escherichia coli strains that are pathogenic to humans. Infect Immun 66, 480-485.

Schubert, S., Fischer, D. \& Heesemann, J. (1999). Ferric enterochelin transport in Yersinia enterocolitica: molecular and evolutionary aspects. J Bacteriol 181, 6387-6395.
Schubert, S., Picard, B., Gouriou, S., Heesemann, J. \& Denamur, E. (2002). Yersinia high-pathogenicity island contributes to virulence in Escherichia coli causing extraintestinal infections. Infect Immun 70, 5335-5337.

Schubert, S., Rakin, A. \& Heesemann, J. (2004). The Yersinia highpathogenicity island (HPI): evolutionary and functional aspects. Int $J$ Med Microbiol 294, 83-94.

Snyder, J. A., Haugen, B. J., Buckles, E. L., Lockatell, C. V., Johnson, D. E., Donnenberg, M. S., Welch, R. A. \& Mobley, H. L. (2004). Transcriptome of uropathogenic Escherichia coli during urinary tract infection. Infect Immun 72, 6373-6381.

Soto, S. M., Smithson, A., Horcajada, J. P., Martinez, J. A., Mensa, J. P. \& Vila, J. (2006). Implication of biofilm formation in the persistence of urinary tract infection caused by uropathogenic Escherichia coli. Clin Microbiol Infect 12, 1034-1036.

Stamm, W. E. \& Norrby, S. R. (2001). Urinary tract infections: disease panorama and challenges. J Infect Dis 183 (Suppl. 1), S1-S4.

Warren, J. W. (2001). Catheter-associated urinary tract infections. Int J Antimicrob Agents 17, 299-303.

Edited by: B. Kenny 